\title{
An Oriented Flux Symmetry Based Active Contour Model for Three Dimensional Vessel Segmentation
}

\author{
Max W.K. Law and Albert C.S. Chung \\ Lo Kwee-Seong Medical Image Analysis Laboratory, \\ Department of Computer Science and Engineering, \\ The Hong Kong University of Science and Technology, Hong Kong \\ \{maxlawwk, achung\}@cse.ust.hk
}

\begin{abstract}
This paper proposes a novel approach to segment three dimensional curvilinear structures, particularly vessels in angiography, by inspecting the symmetry of image gradients. The proposed method stresses the importance of simultaneously considering both the gradient symmetry with respect to the curvilinear structure center, and the gradient antisymmetry with respect to the object boundary. Measuring the image gradient symmetry remarkably suppresses the disturbance introduced by rapid intensity changes along curvilinear structures. Meanwhile, considering the image gradient antisymmetry helps locate the structure boundary. The gradient symmetry and the gradient antisymmetry are evaluated based on the notion of oriented flux. By utilizing the aforementioned gradient symmetry information, an active contour model is tailored to perform segmentation. On the one hand, by exploiting the symmetric image gradient pattern observed at structure centers, the contours expand along curvilinear structures even through there exists intensity fluctuation along the structures. On the other hand, measuring the antisymmetry of the image gradient conveys strong detection responses to precisely drive contours to the structure boundaries, as well as avoiding contour leakages. The proposed method is capable of delivering promising segmentation results. This is validated in the experiments using synthetic data and real vascular images of different modalities, and through the comparison to two well founded and published methods for curvilinear structure segmentation.
\end{abstract}

\section{Introduction}

Segmentation of three dimensional curvilinear objects, particularly vascular structures has a wide range of applications. In the past decades, incorporating curvilinear structurespecific image features in active contour models for vessel segmentation has been intensively studied. For instance, Lorigo et al. [8] developed the CURVES algorithm based on the geodesic active contour model [1], which aims at driving the active contours to the boundaries where image intensity is rapidly changing. The CURVES algorithm employs the minimal curvature regularization term to prevent the evolving contours from vanishing inside narrow vascular structures. Yan and Kassim refined the geodesic active contour model by introducing the capillary force [14] to encourage contours to propagate into small vessels. The contour dynamics of these segmentation methods are

K. Daniilidis, P. Maragos, N. Paragios (Eds.): ECCV 2010, Part III, LNCS 6313, pp. 720-734. 2010.

(C) Springer-Verlag Berlin Heidelberg 2010 
governed by the image intensity gradient. It is possibly problematic if the structure intensity fluctuates along and inside structures. The intensity fluctuation can halt the evolving contours inside structures, and such intensity fluctuation commonly exists in some images, such as angiographic images. Furthermore, low contrast structure boundaries cannot exert enough image force to compete against other forces generated from the intensity fluctuations along the structures. The evolving contours can finally stop inside structures instead of at the boundaries of the structures.

To extract reliable image features for segmentation of three dimensional curvilinear structures, the intensity profiles along the structure cross-sectional plane are commonly considered to be symmetric with respect to the structure center. Classic differential operators, such as the second derivatives of Gaussian [6] and the Hessian matrix [10][4], which are based on convolving an image with symmetric filter functions 1 , were proposed for the detection of curvilinear structures. The differential operators quantify the difference between the intensity inside a local region defined by a scale parameter and those in the vicinity of that local region. Exploiting the Hessian matrix, Toledo et al. [11] developed an active contour model based on the eigenvalues and eigenvectors extracted from the Hessian matrix. In [3], Descoteaux et al. fused the Hessian matrix and the flux measure [12] to formulate an active contour model to segment vascular objects. The flux measure was introduced by Vasilevskly and Siddiqi in [12]. It drives the active contours to segment vessels by using a discretized Laplacian operator, which inspects the intensity changes that occur at the boundary of a local sphere with a predefined radius. Analogous to the original Laplacian operator, the discretized version is isotropic and sensitive to symmetric structures. To handle vessels with various widths, these symmetric operators are always incorporated in multiscale frameworks. However, they commonly return faint responses at structure boundaries. It is because the local intensity variations across the structure boundaries are not symmetric with respect to those boundaries. At the boundaries, the active contours driven by the responses of these operators can evolve randomly according to the image noise attached along the object boundaries. It can lead to subsequent contour leakages.

To segment curvilinear objects such as vessels without leakages, this paper proposes a novel approach that inspects the symmetry of image gradients for active contour evolution. The proposed model considers both the image gradient symmetry with respect to the structure center, and the image gradient antisymmetry with respect to the structure boundary. Analyzing both the gradient symmetry and antisymmetry helps devise image features to encourage contour propagation even through there exists intensity fluctuation along structures, and simultaneously avoids contour leakages. In this paper, through the experiments using a noise corrupted synthetic image volume and real vascular image volumes, the proposed method is compared with two well founded published approaches, the flux method [12] and the CURVES algorithm [8]. The ability of the proposed method to correctly segment curvilinear structures, particularly vasculatures without leakages is validated. It consistently delivers promising segmentation results in all cases. It is therefore well suited to perform segmentation of curvilinear structures.

\footnotetext{
${ }^{1}$ The image Hessian matrices can be found by convolving the image with a set of the second derivatives of Gaussian [10].
} 


\section{Methodology}

Without the loss of generality, we assume that the objects of interest have stronger intensity than image backgrounds. For the detection of curvilinear structures, we first analyze the image gradients at a local spherical region boundary which touches the object boundary. These image gradients are projected along a direction on the object's cross-sectional plane. As shown in Fig. 1a, along the cross-sectional plane of a curvilinear structure, the image gradients point to the structure center and form a symmetric pattern. When the local spherical region centers at the middle of the object, the projected gradients are symmetric (see Fig. 1b). The symmetry of the projected gradients implies that both the magnitudes and the orientations of the projected gradients are symmetric with respect to the spherical region center. When the local spherical region centers at other positions, the projected gradients are aligned in various patterns (see Figs. 11-e). At the object boundary, the projected gradients at the local spherical region boundary point along the same direction (Fig. 1 d). As such, the projected gradient magnitudes are symmetric but the projected gradient orientations are antisymmetric with respect to the spherical region center. This pattern of image gradients is referred to as the antisymmetric pattern (Fig.11d). In the positions slightly inside or outside the structure, the projected image gradients are similar to the patterns as shown in Figs. 1t and e respectively, in which both the projected gradient magnitudes and orientations are antisymmetric. This pattern of projected gradients is considered as neither symmetric nor antisymmetric. In summary, there are three situations discussed regarding various positions located,

- at the structure centers, the projected gradients are symmetric (the projected gradient magnitudes and orientations are symmetric);

- at object boundaries, the projected gradients are antisymmetric (the projected gradient magnitudes are symmetric but their orientations are antisymmetric); and

- slightly inside or outside the object, the projected gradients are neither symmetric nor antisymmetric.

\subsection{Oriented Flux Symmetry}

In this section, two measures are devised to analyze the symmetric gradient patterns and the antisymmetric gradient patterns. These two measures jointly quantify the gradient symmetry. They are therefore conveying reliable detection responses to identify the aforementioned three situations. This detection scheme is referred to as oriented flux symmetry. In oriented flux symmetry detection scheme, the first measure to help quantify the gradient symmetry is introduced on the basis of a previous work, called optimally oriented flux [7]. The oriented flux measure quantifies the amount of image gradient, which is projected on a direction $\hat{\rho}$, flowing into a local spherical region centered at $\boldsymbol{x}$

$$
f(\boldsymbol{x} ; r, \hat{\rho})=\frac{1}{4 \pi r^{2}} \int_{\partial B_{r}}((\boldsymbol{v}(\boldsymbol{x}+\boldsymbol{A}) \cdot \hat{\rho}) \hat{\rho}) \cdot \hat{n} d A,
$$

where $B_{r}$ is a local spherical region with radius $r, \boldsymbol{A}$ is the position vector on $\partial B_{r}$, $\hat{n}$ is the inward normal of the sphere at $\boldsymbol{A}, d A$ is the infinitesimal area on $\partial B_{r}$ and $\boldsymbol{v}$ is image gradient. The differentiability of a discrete image can be approximated by 
Table 1. The analysis of the response magnitudes of various measures obtained at different positions $\boldsymbol{x}$. In the second, the third and the fifth columns, $\hat{\rho}$ is given as the direction on the structure cross-sectional plane, pointing from object centers to $\boldsymbol{x}$. In the second to the fifth columns except the entries with ${ }^{*}, r$ is given as the distance from $\boldsymbol{x}$ to the nearest object boundary; in the entries with $^{*}, r$ is assumed to be a value smaller than the structure radius.

\begin{tabular}{|c|c|c|c|c|c|}
\hline Location of $\boldsymbol{x}$, relative to a curvilinear object & $f(\boldsymbol{x} ; r ; \hat{\rho})$ & $s(\boldsymbol{x} ; r ; \hat{\rho})$ & $\Lambda_{12}(\boldsymbol{x} ; r)$ & $Q_{12}(\boldsymbol{x} ; r)$ & $M(\boldsymbol{x})$ \\
\hline At the center (Fig. $1 \mathrm{~b}$ ) & $>>0$ & $\approx 0$ & $>>0$ & $\approx 0$ & $>>0$ \\
\hline At the boundary (Fig. 11) & $\approx 0$ & $>>0$ & $\approx 0$ & $>>0$ & $=0$ \\
\hline Slightly inside or outside the object (Figs. $1 \mathrm{f}$ and e) & $>0$ & $>0$ & $>0$ & $>0$ & $\approx 0$ \\
\hline
\end{tabular}

obtaining the image gradient from the image smoothed by a Gaussian filter with the scale factor of 1 .

Whereas the authors of [7] focused on finding the optimal projection orientation to maximize the resultant value of the oriented flux measure, we aim at making use of the above equation to help quantify the image gradient symmetry. In this aspect, the oriented flux is regarded as a measure sensitive to the symmetric image gradient pattern. The above oriented flux measure detects curvilinear structures grounded on its high sensitivity to the symmetric gradient pattern, as shown in Fig. $1 \mathrm{~b}$. Given that Equation 1 is evaluated when $\hat{\rho}$ is a direction on the structure cross-sectional plane and $r$ is equal to the structure radius, $f(\cdot)$ attains its maximal value at the structure centers. The gradient symmetry decreases with respect to the positions away from the centers and thus, the strength of the oriented flux detection response declines accordingly. To identify the antisymmetric gradient pattern, the second measure is devised as,

$$
s(\boldsymbol{x} ; r, \hat{\rho})=\frac{1}{4 \pi r^{2}} \int_{\partial B_{r}}(\boldsymbol{v}(\boldsymbol{x}+\boldsymbol{A}) \cdot \hat{\rho}) d A .
$$

This measure helps quantify the antisymmetry of the image gradients that contributes to the resultant value of the above oriented flux measure (Equation 1). It is referred to as oriented flux antisymmetry (OFA). It is sensitive to antisymmetric gradient patterns occurring at object boundaries. The OFA measure and the oriented flux measure alternatively return strong detection responses at the structure centers and at the structure boundaries (see the second and the third columns of Table 1 ).

\subsection{Quantifying Gradient Symmetry along Structure Cross-Sectional Planes}

Developing a measure to indicate the middle of vascular structures is now possible by aggregating the OFA measure and the oriented flux measure. It is achieved by first performing the eigen decomposition on a tensor to obtain the optimal projection axis which maximizes the magnitude of the oriented flux measure [7]. There are three pairs of resultant eigenvalues and eigenvectors, denoted as $\lambda_{j}(\boldsymbol{x} ; r)$ and $\hat{e}_{j}(\boldsymbol{x} ; r)$ respectively, where $j \in[1,2,3],\left|\lambda_{1}(\cdot)\right| \geq\left|\lambda_{2}(\cdot)\right| \geq\left|\lambda_{3}(\cdot)\right|$. To detect curvilinear structures, the amount of the image gradients pointing to the structure center along its cross-sectional plane spanned by $\hat{e}_{1}(\cdot)$ and $\hat{e}_{2}(\cdot)[7]$ is evaluated, 


$$
\begin{aligned}
\Lambda_{12}(\boldsymbol{x} ; r) & =\frac{1}{4 \pi r^{2}} \int_{\partial B_{r}}\left(\left[\hat{e}_{1}(\boldsymbol{x} ; r) \hat{e}_{2}(\boldsymbol{x} ; r)\right]^{T} \boldsymbol{v}(\boldsymbol{x}+\boldsymbol{A})\right) \cdot\left(\left[\hat{e}_{1}(\boldsymbol{x} ; r) \hat{e}_{2}(\boldsymbol{x} ; r)\right]^{T} \hat{n}\right) d A \\
& =f\left(\boldsymbol{x} ; r, \hat{e}_{1}(\boldsymbol{x}, r)\right)+f\left(\boldsymbol{x} ; r, \hat{e}_{2}(\boldsymbol{x}, r)\right)=\lambda_{1}(\boldsymbol{x} ; r)+\lambda_{2}(\boldsymbol{x} ; r) .
\end{aligned}
$$

Utilizing Equation 2] an OFA based measure associated with $\hat{e}_{1}(\cdot)$ and $\hat{e}_{2}(\cdot)$ is used to inspect the antisymmetry of gradients along structure cross-sectional planes,

$$
\begin{aligned}
Q_{12}(\boldsymbol{x} ; r) & =\frac{1}{4 \pi r^{2}}\left|\int_{\partial B_{r}}\left(\left[\hat{e}_{1}(\boldsymbol{x} ; r) \hat{e}_{2}(\boldsymbol{x} ; r)\right]^{T} \boldsymbol{v}(\boldsymbol{x}+\boldsymbol{A})\right) d A\right| \\
& =\sqrt{s^{2}\left(\boldsymbol{x} ; r, \hat{e}_{1}\right)+s^{2}\left(\boldsymbol{x} ; r, \hat{e}_{2}\right)} .
\end{aligned}
$$

The above equation evaluates the magnitude of the sum of the projected image gradients at $\partial B_{r}$ on the detected structure cross-sectional plane. A moderate or large resultant value signals the situation that $\boldsymbol{x}$ is not located at the structure center (see the fifth column in Table 1). As presented in the second row, the fourth and the fifth columns in Table 1 $\Lambda_{12}(\cdot)>>Q_{12}(\cdot)$ in the middle of a curvilinear structure. Also, both $Q_{12}(\cdot)$ and $\Lambda_{12}(\cdot)$ are robust against the intensity fluctuation along structure because they are evaluated along its cross-sectional planes. Besides, $\Lambda_{12}(\cdot)$ cannot give a very large magnitude outside the middle of structures (see the fourth column in Table 1), including the positions either inside the structures and closed to the structure boundaries, at the boundaries, or slightly outside the structure. It is because the gradients are not symmetric at these positions. Based on $\Lambda_{12}(\cdot)$ and $Q_{12}(\cdot)$, a measure that only reports positive responses in the middle of structure is, $\max \left(0, \Lambda_{12}(\boldsymbol{x} ; r)-Q_{12}(\boldsymbol{x} ; r)\right)$. As the target object radius is unknown, the detection response at $\boldsymbol{x}$ is the maximum response among those responses computed in a set of radii. It therefore retrieves the most significant responses induced by the image gradients located at the object boundaries. As such,

$$
M(\boldsymbol{x})=\max _{r \in R}\left(\max \left(0, \Lambda_{12}(\boldsymbol{x} ; r)-Q_{12}(\boldsymbol{x} ; r)\right)\right) .
$$

$R$ is the radius set and is specified to include all possible radii of the target structures.

Regarding the proposed active contour based segmentation algorithm, the measure $M(\boldsymbol{x})$ guides the evolving contours to expand along and inside curvilinear structures, even through there exists intensity fluctuation along them. To illustrate this idea, $M(\boldsymbol{x})$ is evaluated using a noise corrupted synthetic tube with a radius of 4 voxels (Figs. 2 ad). In this example, the radius set for $M(\cdot)$ is specified as $R=\{1,1.5,2, \ldots 6\}$ voxels. A sharp intensity drop is observed along the tube. This synthetic tube exaggerates the situation where a sudden intensity change is present along a structure. Many existing active contour approaches [1][8][14] can misidentify sudden intensity drops as parts of object boundaries. On the contrary, the measure $M(\boldsymbol{x})$ can consistently deliver positive detected values in the middle of the synthetic tube despite the intensity drop (Fig. 22). In each sub-figure of Fig. 2], it is observed when the detection radius of $M(\boldsymbol{x})$ differs from the structure radius (all cases, except the one with " $R=\{4\}$ "), the detection responses are smaller than that with a matched radius (in the case of " $R=\{4\}$ "). It is because the symmetric gradient pattern vanishes as the spherical region radius differs from the 


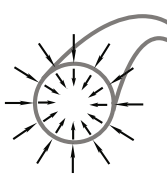

(a)

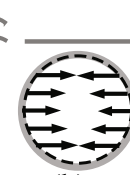

(b)

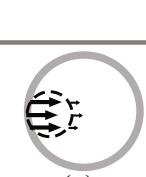

(c)

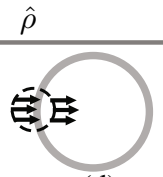

(d)

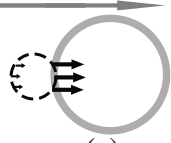

(e)

Fig. 1. Illustrations of image gradients which form various patterns. The black arrows and grey solid lines represent image gradients and structure boundaries respectively. (a) Image gradients along a curvilinear structure cross-sectional plane. (b-e) Four examples showing image gradients located at the local spherical region boundaries (black dotted circles), projected along $\hat{\rho}$.

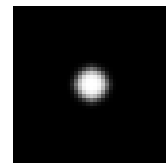

(a)

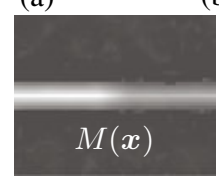

(e)

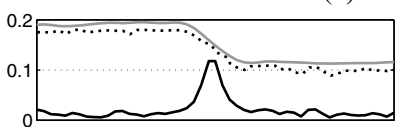

(h)
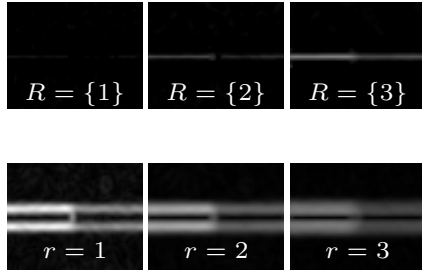

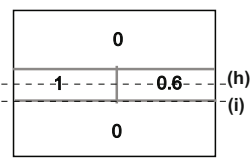

(c)

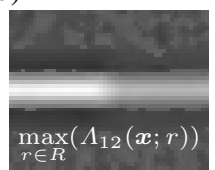

(f)

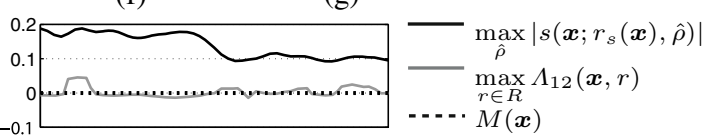

(i)

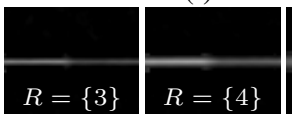

(j)
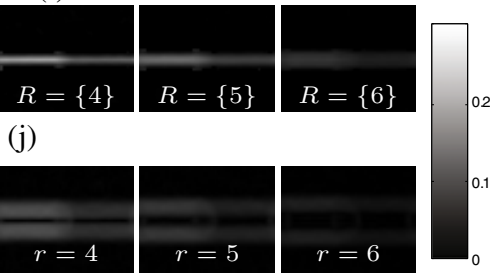

(k)

Fig. 2. (a) An xy-plane which shows the cross-section of the synthetic tube with a 4 voxel radius. (b) An xz-plane of the synthetic tube. (c) The numbers represent the intensity of various parts of the image in (b). (d) An xz-plane of the synthetic tube corrupted by additive Gaussian noise with standard deviation 0.1. (e-g) The xz-planes which shows different measures. The black line in (g) showing the boundary where $\max _{\hat{\rho}}\left|s\left(\boldsymbol{x} ; r_{s}(\boldsymbol{x}), \hat{\rho}\right)\right|$ is maximal along the vertical directions from the tube center to the image background. (h-i) The profiles of different measures obtained along the lines shown as dotted lines in (c). (j-k) The values of $M(\boldsymbol{x})$ and $\max _{\hat{\rho}}|s(\boldsymbol{x} ; r, \hat{\rho})|$, which are obtained using one radius for each sub-figure. 


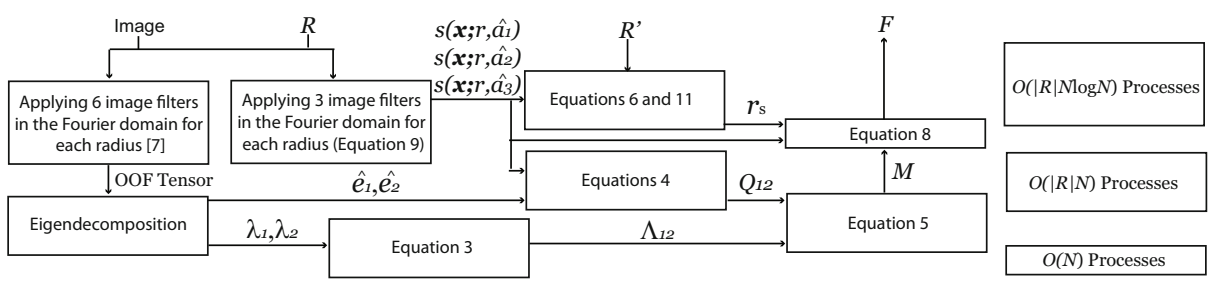

Fig. 3. The flow chart of the efficient algorithm to retrieve the level set evolution speed function $F(\boldsymbol{x})$ (Equation 8 ). The block sizes vary according to the complexity of different tasks.

structure radius. Thus, acquiring the maximum response obtained among all radii as in Equation 5 offers a reliable measure to quantify the symmetric gradient patterns.

As a major component of $M(\boldsymbol{x})$, the magnitude of $\Lambda_{12}(\cdot)$ is insignificant at object boundaries (see the third row, the fourth column in Table 1). This observation is validated using the above synthetic tube (see the grey solid line in Fig. 2], and it is a plot along the dotted line (i) in Fig. 2r). Along the tube boundary, the response magnitude of $\Lambda_{12}(\cdot)$ is small and slightly fluctuating along the tube boundary. The response magnitude exhibits no significant change at the position where the tube intensity drops from 1.0 to 0.6 . This implies that the response fluctuates randomly instead of following the tube intensity. Returning faint and randomly fluctuating response magnitudes along object boundaries is common to the approaches that extract image features by using symmetric measures, such as the oriented flux measure, the Hessian matrix [4][11][3] and the discretized Laplacian operator used by the flux method [12]. Since the local intensity variations across the object boundaries are not symmetric with respect to the boundaries, these symmetric measures deliver noisy responses at object boundaries. Evolving an active contour according to the symmetric measure based responses can lead to subsequent contour leakages. In the proposed method, $M(\cdot)$ is obtained by subtracting $\Lambda_{12}(\cdot)$ from $Q_{12}(\cdot)$. It keeps the resultant values of $M(\cdot)$ zero at the object boundaries (see in the third row, the fourth to the sixth columns in Table 1). It avoids the interference in the detection results incurred by the fluctuating responses of $\Lambda_{12}(\cdot)$ along boundaries.

\subsection{The Oriented Flux Symmetry Based Active Contour Model}

To locate the structure boundaries in the proposed active contour model, the OFA measure which can capture the antisymmetric gradient patterns occurring at object boundaries is utilized. Suppose that $\mathcal{C}$ is a closed contour and $\mathcal{N}$ is the contour inward normal, one of the criteria of finding the desired segmentation solution is to maximize $\int_{\mathcal{C}} s(\mathcal{S} ; r, \mathcal{N}) d S$, where $\mathcal{S}$ and $d S$ are the position vector and the infinitesimal area on $\mathcal{C}$ respectively. Regarding the value of $r$, for positions inside curvilinear structures or slightly outside the structures, a proper value is the distance from those positions to the closest object boundary. It ensures that the responses of $s(\cdot)$ computed at the various positions, such as those shown in Figs. 1k and e are significant and produced by the image gradient at the object boundaries. It is illustrated in Fig. 2k k, when $r$ is small (1 or 2 voxels), the OFA responses are concentrated in the vicinity of the tube boundary. 
As $r$ grows, more OFA responses can be observed in the regions further away from the tube boundary, despite the generally weaker responses than those obtained using smaller values of $r$. Therefore, large values of $r$ can guide the evolving contours which are located further away from object boundaries. Meanwhile, a small valued $r$ is beneficial to precisely indicate the boundaries. Hence, $r$ is estimated at each location by observing the OFA measure along the direction giving the strongest detection response, which is maximal among a set of radii,

$$
r_{s}(\boldsymbol{x})=\arg \max _{r \in R^{\prime}}\left(\max _{\hat{\rho}}|s(\boldsymbol{x} ; r, \hat{\rho})|\right)=\arg \max _{r \in R^{\prime}}\left|\frac{1}{4 \pi r^{2}} \int_{\partial B_{r}} \boldsymbol{v}(\boldsymbol{x}+\boldsymbol{A}) d A\right| .
$$

To recognize structures adjacent to the strong edges of undesired objects, $R^{\prime}$ can contain only the smallest radius in $R$ discussed in the previous section. This avoids the detection being adversely affected by the strong edges of adjacent objects. For detection of curvilinear structures with complicated geometry (e.g. high curvature vessel or bifurcation) or irregular cross-sections, $R^{\prime}$ can be defined as the same as $R$. It ensures that various positions inside or slightly outside the structures can reach the nearest object boundary by those radii in $R$. In Fig. $2 \mathrm{~g}$, the value of $\max _{\hat{\rho}}\left|s\left(\boldsymbol{x} ; r_{s}(\boldsymbol{x}), \hat{\rho}\right)\right|$ is presented. Its profiles along the dotted lines in Fig. 2re are given in Figs. 2h and i. Along the vertical direction in Fig. $2 \mathrm{~g}$, from the tube center to the image background regions (in the upper half and in the lower half of Fig. $2 \mathrm{~g})$, the locations where $\max _{\hat{\rho}}\left|s\left(\boldsymbol{x} ; r_{s}(\boldsymbol{x}), \hat{\rho}\right)\right|$ attains its maximum are shown as two black lines. These black lines are located along the tube boundaries, which become distinctive in the image of $\max _{\hat{\rho}}\left|s\left(\boldsymbol{x} ; r_{s}(\boldsymbol{x}), \hat{\rho}\right)\right|$. It illustrates that evolving contours according to the OFA measure with the detection radius $r_{s}(\boldsymbol{x})$ can facilitate the detection of object boundaries.

The OFA measure is not limited to the detection of curvilinear structures unlike the oriented flux measure does. The OFA measure can also highlight the boundaries of various kinds of structures, which deviates from the curvilinear ones. However, this flexibility implies that the OFA measure is sensitive to all intensity changes, including the intensity fluctuation along curvilinear structures. As shown by the black solid line in Fig. 2h, a large value of $\max _{\hat{\rho}}\left|s\left(\boldsymbol{x} ; r_{s}(\boldsymbol{x}), \hat{\rho}\right)\right|$ is observed when the tube intensity drops from 1.0 to 0.6 inside the synthetic tube. Nonetheless, $M(\boldsymbol{x})$ retains a high detection response as compared to $\max _{\hat{\rho}}\left|s\left(\boldsymbol{x} ; r_{s}(\boldsymbol{x}), \hat{\rho}\right)\right|$ (see the black solid line and the black dotted line in Fig. 2h). On the contrary, $\max _{\hat{\rho}}\left|s\left(\boldsymbol{x} ; r_{s}(\boldsymbol{x}), \hat{\rho}\right)\right|$ is large at the tube boundary as compared to $M(\boldsymbol{x})$ (see the black solid line and black dotted line in Fig. 2i). These two measures alternatively deliver higher responses than their counterparts at the structure centers and at the object boundaries (also see the last two columns of Table 1). Hence, the desired resultant contour maximizes the following energy functional,

$$
E(\mathcal{C})=\int_{\operatorname{Inside}(\mathcal{C})} M(\mathcal{V}) d V+\int_{\mathcal{C}} s\left(\mathcal{S} ; r_{s}(\mathcal{S}), \mathcal{N}\right) d S
$$

where $\mathcal{V}$ and $d V$ are the position vector and the infinitesimal volume respectively. The evolving contour $\mathcal{C}$ is represented as the zero level of a level set function $\phi$ [9]. By using the gradient descent approach, the dynamic of the level set function is described 
as $\phi_{t}=F|\nabla \phi|$, where $F$ is the first variation of $E(\mathcal{C})$, i.e.

$$
F(\boldsymbol{x})=M(\boldsymbol{x})-\operatorname{div}\left(\frac{1}{4 \pi r_{s}^{2}(\boldsymbol{x})} \int_{\partial B r_{s}(\boldsymbol{x})} \boldsymbol{v}(\boldsymbol{x}+\boldsymbol{A}) d A\right) .
$$

Considering the large positive responses of $M(\boldsymbol{x})$ in the middle of curvilinear structures, the regions with large values of $M(\boldsymbol{x})$ can be used as the seed positions to initialize the contour evolution. The function $F(\boldsymbol{x})$ is positive inside curvilinear structures to keep the contour expanding. It is negative at the positions slightly outside the structure boundaries. This eventually stops the evolving contour over the structure boundaries.

\subsection{Fourier Expressions of the Oriented Flux Measure and the Oriented Flux Antisymmetry Measure}

Studying the Fourier expressions helps devise the efficient computation algorithm for the proposed measures. It also reveals the orthogonality of the oriented flux measure and the OFA measure if they are regarded as two types of image filters. Denote FFT be the fast Fourier transform operator, i.e. $F F T^{-1}\{F F T\{I\}\} \equiv I$ and $\boldsymbol{u}$ is the frequency (in cycle per millimeter). The Fourier expression of the OFA measure $s(\boldsymbol{x} ; r, \hat{\rho})$ can be found by first rewriting Equation 2 as,

$$
s(\boldsymbol{x} ; r, \hat{\rho})=\int_{\text {Image }} D_{r}(\boldsymbol{V})(\hat{\rho} \cdot(\boldsymbol{\nabla} g) * I)(\boldsymbol{x}+\boldsymbol{V}) d V=\left(\left(\hat{\rho} \cdot(\boldsymbol{\nabla} g) * D_{r}\right)(\boldsymbol{x})\right) * I(\boldsymbol{x}),
$$

where $g$ is the Gaussian filter employed for smoothing the input image as discussed in Section 2.1 and $D_{r}(\boldsymbol{x})$ is a spherical impulse function which is equal to $\left(4 \pi r^{2}\right)^{-1}$ when $\|\boldsymbol{x}\|=r$ and 0 elsewhere. By employing the Hankel transform,

$$
\operatorname{FFT}\left\{\left(\hat{\rho} \cdot(\boldsymbol{\nabla} g) * D_{r}\right)\right\}=\sqrt{-1}(r\|\boldsymbol{u}\|)^{-1}(\hat{\rho} \cdot \boldsymbol{u}) e^{-2(\pi\|\boldsymbol{u}\|)^{2}} \sin (2 \pi r\|\boldsymbol{u}\|) .
$$

Besides, as stated in [7], $f(\boldsymbol{x} ; r, \hat{\rho})$ can be computed by

$$
F F T^{-1}\left\{F F T\{I\}\left(\frac{4 \pi r\left(\hat{\rho}\left(\boldsymbol{u}^{T} \boldsymbol{u}\right) \hat{\rho}^{T}\right)}{\|\boldsymbol{u}\|^{2} e^{2(\pi\|\boldsymbol{u}\| \sigma)^{2}}}\right)\left(\cos (2 \pi r\|\boldsymbol{u}\|)-\frac{\sin (2 \pi r\|\boldsymbol{u}\|)}{2 \pi r\|\boldsymbol{u}\|}\right)\right\} .
$$

As such, the computation of the oriented flux measure and the OFA measure are considered as two filtering operations. To facilitate the discussion, we denote $\Phi_{\hat{\rho}, r}(\boldsymbol{u})=$ $F F T\left\{\left(\hat{\rho} \cdot(\nabla g) * D_{r}\right)\right\}$, and the non-image terms in Equation 10 (i.e. the terms after $F F T(I))$ as $\Psi_{\hat{\rho}, r}(\boldsymbol{u})$. These two functions exhibit the following properties,

$\Phi_{\hat{\rho}, r}(-\boldsymbol{u}) \Psi_{\hat{\rho}, r}(-\boldsymbol{u})=-\Phi_{\hat{\rho}, r}(\boldsymbol{u}) \Psi_{\hat{\rho}, r}(\boldsymbol{u}), \lim _{\boldsymbol{u} \rightarrow \mathbf{0}} \Phi_{\hat{\rho}, r}(\boldsymbol{u})=\lim _{\boldsymbol{u} \rightarrow \mathbf{0}} \Psi_{\hat{\rho}, r}(\boldsymbol{u})=0$, and thus, $\int_{\text {Image }}\left(F F T^{-1}\left\{\Phi_{\hat{\rho}, r}\right\}(\boldsymbol{x})\right)\left(F F T^{-1}\{\Psi\}^{*}(\boldsymbol{x})\right) d \boldsymbol{x}=\int_{\text {Image bandwidth }} \Phi_{\hat{\rho}, r}(\boldsymbol{u}) \Psi_{\hat{\rho}, r}(\boldsymbol{u}) d \boldsymbol{u}=0$,

\footnotetext{
${ }^{2}$ The implementation is based on [13] and a publicly available library, "The Insight Segmentation and Registration Toolkit" (http://www.itk.org). The level set evolution is stopped when the increment of the segmented voxels over 20 iterations is less than $0.01 \%$ of them.
} 


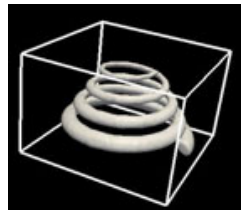

(a)

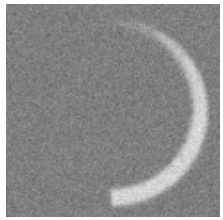

(b)

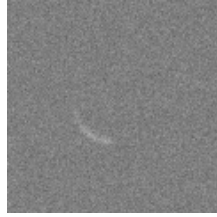

(c)

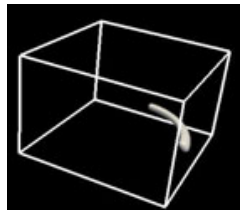

(d)

Fig. 4. The $80 \times 80 \times 80$ voxels synthetic image used in the synthetic data experiment. (a) the isosurface of the spiral with the isovalue of 0.5 ; (b) the 15th slice showing the bottom part of the noise corrupted spiral; (c) the 65th slice showing the top part of the noise corrupted spiral; (d) the initial level set function for the segmentation of the spiral.

where the superscript $*$ is function conjugate. Hence, given the same radius $r$ and orientation $\hat{\rho}$, the oriented flux measure and the OFA measure can be regarded as two orthogonal image filters. They convey two distinct types of information - the gradient symmetry and the gradient antisymmetry. Fusing this information, the measure $M(\boldsymbol{x})$ (Equation 5) judges which type of the information is more significant at a given position. It delivers responses only if that position exhibits a greater degree of gradient symmetry than that of antisymmetry.

On the other hand, the level set evolution speed $F(\boldsymbol{x})$ is independent of the evolving contour. It is therefore evaluated prior to the level set evolution process, in which,

$$
\left[s\left(\boldsymbol{x} ; r, \hat{a}_{1}\right) s\left(\boldsymbol{x} ; r, \hat{a}_{2}\right) s\left(\boldsymbol{x} ; r, \hat{a}_{3}\right)\right]^{T}=\frac{1}{4 \pi r^{2}} \int_{\partial B r} \boldsymbol{v}(\boldsymbol{x}+\boldsymbol{A}) d A,
$$

where $\hat{a_{1}}, \hat{a_{1}}$ and $\hat{a_{3}}$ are the unit vectors along the $\mathrm{x}-, \mathrm{y}$ - and $\mathrm{z}$-directions. With the aid of the aforementioned Fourier expressions, $F(\boldsymbol{x})$ is evaluated efficiently, with complexity $O(|R| N \log N)$. This is summarized in Fig. 3 It is noted that, whereas the complicated formulation of $F(\boldsymbol{x})$, its complexity is comparable to that of the FFT-based multiscale Hessian techniques (see [7] for details). Finally, the divergence in Equation 8 is evaluated using the central difference scheme.

\section{Experimental Results}

The proposed method is compared with two published vascular segmentation techniques, the CURVES algorithm [8] (CURVES) and the flux method [12] (FLUX). Prior to performing segmentation using these methods, the image volumes are smoothed by a Gaussian filter with a scale factor of 1 smallest voxel length, for noise reduction for CURVES and FLUX, and for ensuring differentiability of the discrete image signal for the proposed method. Based on visual assessments of the clinical data, the widths of the target structures are all less than $3 \mathrm{~mm}$. The radius set used for FLUX and the proposed method $R$ covers the radii from 1 voxel-length (the physical length depends on the voxel sizes of different images) to $3 \mathrm{~mm}$ (or 8 voxel-length in the synthetic case). The second radius set for the proposed method $R^{\prime}$ is the same as $R$ in all tests except the fourth real vascular image case. For CURVES, for each case, we present the structure which reports no leakage and that segmented region has the largest number of segmented voxels among those obtained using different heuristic parameter values used by CURVES. 


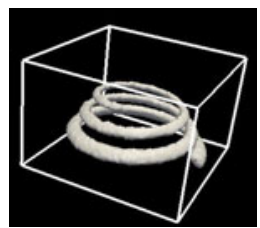

CURVES

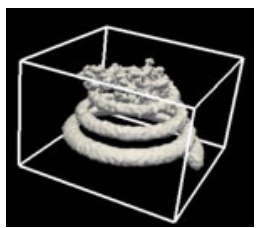

FLUX

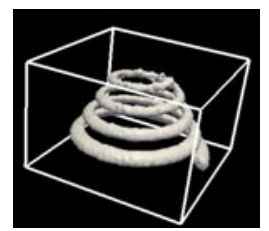

The proposed method

Fig. 5. The segmentation results of the noise corrupted synthetic spiral by using CURVES, FLUX and the proposed method
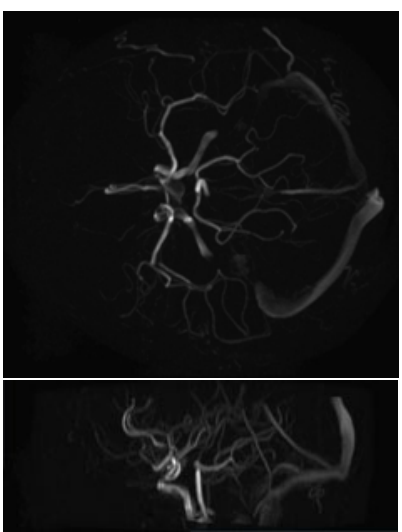

Image dimension: $512 \times 512 \times 64$ voxels Voxel size: $0.39 \times 0.39 \times 1.0 \mathrm{~mm}^{3}$

(a)
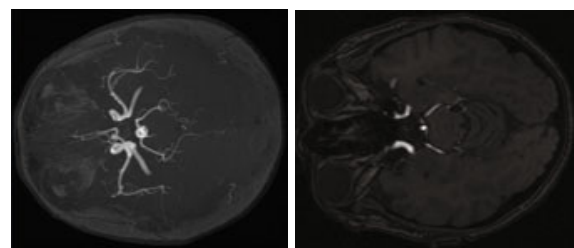

Image dimension: $512 \times 512 \times 60$ voxels Voxel size: $0.39 \times 0.39 \times 0.95 \mathrm{~mm}^{3}$

(c)

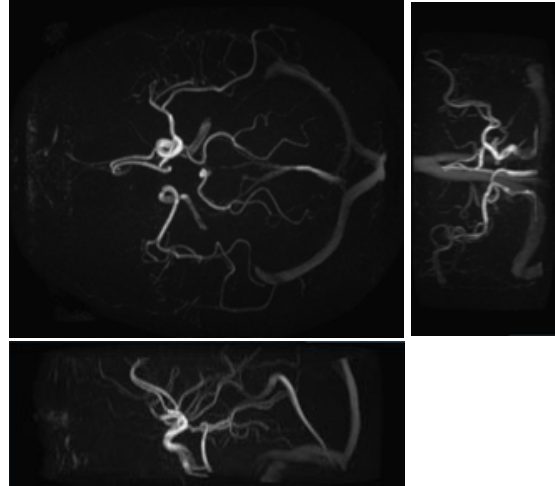

Image dimension: $512 \times 512 \times 64$ voxels Voxel size: $0.39 \times 0.39 \times 1.0 \mathrm{~mm}^{3}$

(b)
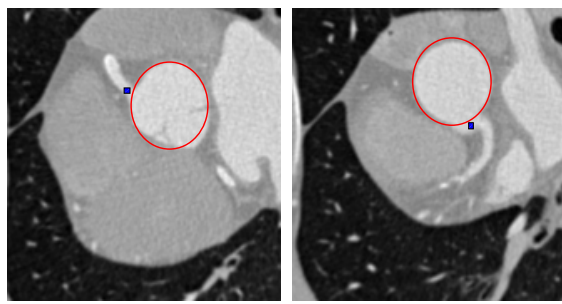

Image dimension: $380 \times 360 \times 236$ voxels Voxel size: $0.32 \times 0.32 \times 0.40 \mathrm{~mm}^{3}$

(d)

Fig. 6. The image volumes used in the real vascular image experiment. (a, b) The perspective maximum intensity projections, along the axial, the sagittal and the coronal directions of two intracranial PC-MRA volumes; (c) the axial perspective maximum intensity projection (left) and the 53th image slice (right) of an intracranial TOF-MRA volume; (d) The 182th (left) and 214th (right) slices of a cardiac CTA volume. The red circles indicate the aorta and the blue dots are the manually placed initial seed points. (Please refer to the electronic version of this paper for better illustration). 


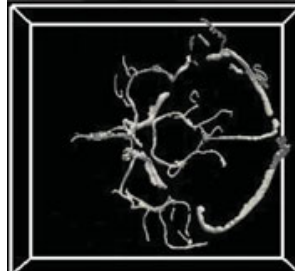

(a)

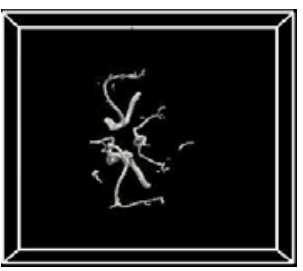

(e)

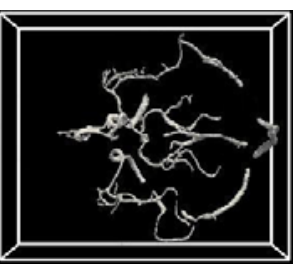

(b)

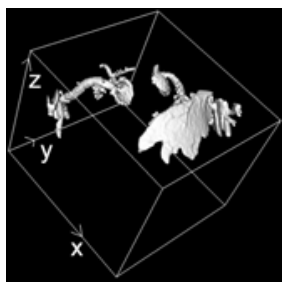

(f)

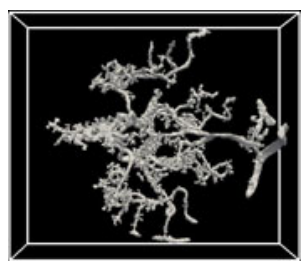

(c)

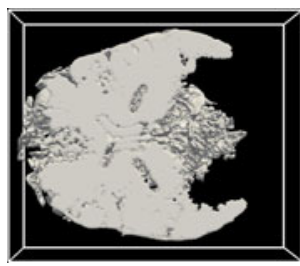

(g)

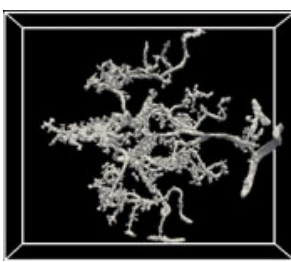

(d)

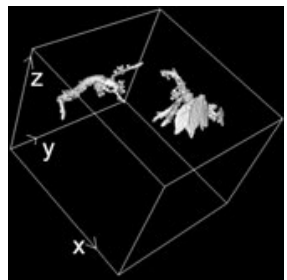

(h)

Fig. 7. (a, b, e, f) The segmentation results of the clinical cases shown in Figs. 6a, b, c and d respectively, by using CURVES. (c, d, g, h) The segmentation results of the clinical cases shown in Figs. 6a, b, c and d respectively, by using FLUX.

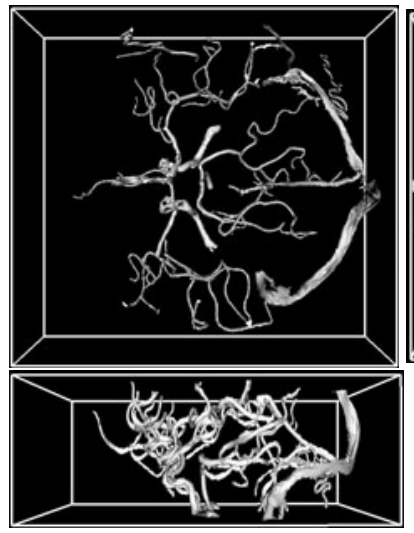

(a)

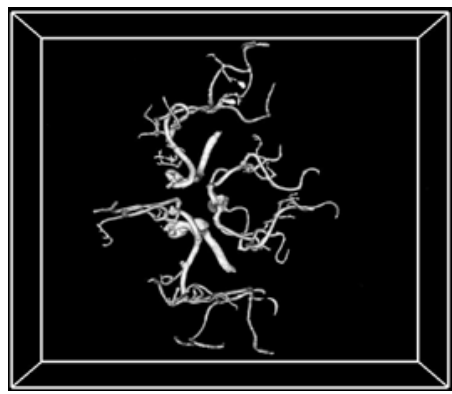

(c)
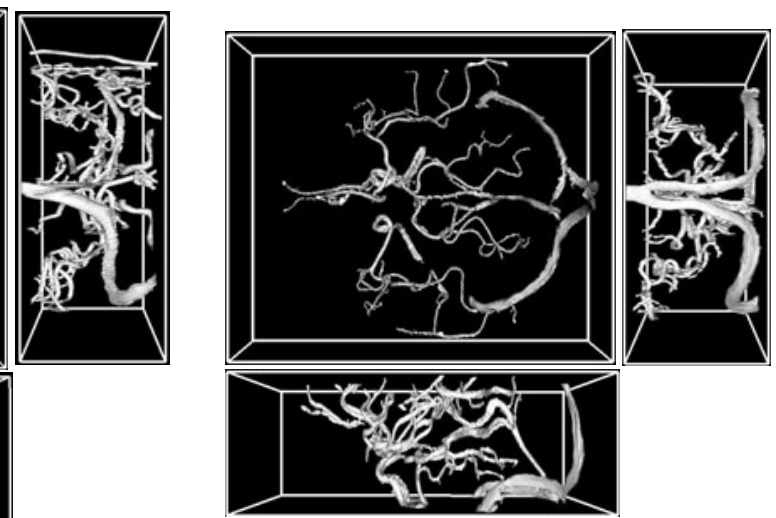

(b)

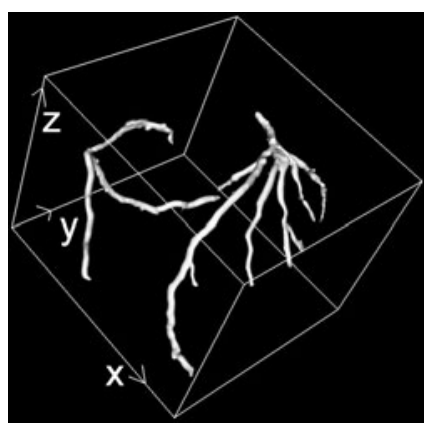

(d)

Fig. 8. The segmentation results obtained by using the proposed method from the four angiographic images shown in Figs. 6a-d 


\subsection{Synthetic Data}

Using a noise corrupted synthetic spiral, as shown in Figs. 4ha-c, we examine the ability of different approaches to segment an elongated structure, where the intensity is changing along the structure and image noise is present. The inner radius and the intensity of the spiral are gradually reduced from 4 voxels and a value of 1 at the bottom of the structure, to 1 voxel and a value of 0.5 at the top of the structure. This synthetic spiral is corrupted using additive Gaussian noise with standard deviation equal to 0.1.

The active contours of all methods are initialized inside the bottom part of the spiral (Fig. 4d). In Fig. 5, the segmentation results of various approaches are shown. In which, the contour of CURVES cannot propagate along the spiral to reach the top of the structure. As the image intensity declines along the structure, the image gradient generated by the image noise inside the spiral exerts higher image force than that exerted by the weak boundary of the structure having low intensity value. Thus, the evolving contour of CURVES is halted inside the structure. Besides, the contour of FLUX (Fig. 5) penetrates the object boundary and it results in contour leakages. It is because the symmetric discretized Laplacian operator used by FLUX returns faint responses along object boundaries. The contour is randomly evolved at the low contrast spiral boundaries and leaks through these boundaries. On the contrary, for the proposed method, the measure $M(\boldsymbol{x})$ allows the contour to propagate along structure and the OFA measure stops the evolving contour at object boundaries. The proposed method is therefore capable of segmenting the entire spiral without leakages (see Fig. 5, the proposed method).

\subsection{Real Vascular Images}

There are four angiographic images employed in this experiment, including two intracranial phrase contrast magnetic resonance angiographic (PC-MRA) images ${ }^{3}$ (Figs. 6 6 and b), one intracranial time-of-flight MRA (TOF-MRA) image 3 (Fig. 6r) and one cardiac computed tomographic angiographic (CTA) image 4 (Fig. 6d). The voxel intensity of these images was scaled to be in the range of 0 and 1 . The experimental settings of different approaches are the same as those settings in the synthetic data experiments except the procedures of contour initialization. For the PC-MRA and TOF-MRA image volumes, the initial level set function is obtained by thresholding the $0.1 \%$ image voxels, which produce the highest values of $M(\boldsymbol{x})$ among all voxels in the image. The initial contours are only placed in the middle of vessels with large detected values of $M(\boldsymbol{x})$. For the CTA image volume, the object of interest - coronary arteries are connected with the aorta, which is not a part of the target region. They share the same intensity range. We manually select two spheres with a radius of $3 \mathrm{~mm}$ at two positions where the aorta is connected with the left coronary artery, and the right coronary artery. The level set update (i.e. the contour evolution) is disabled within these two spheres for all methods. Two initial seeds are placed in the left coronary artery and the right coronary artery (see the blue dots in Fig. (6d). In this CTA image, the radius set $R^{\prime}$ of the proposed method

\footnotetext{
${ }^{3}$ Acquired using a Philips 3T ACS Gyroscan MR scanner without the use of contrast agent, at the University Hospital of Zurich, Switzerland.

${ }^{4}$ Rotterdam Coronary Artery Algorithm Evaluation Framework, http: / / coronary.bigr.nl/
} 
contains only the smallest radius in $R$ to avoid the disturbance introduced by the edges adjacent to the arteries (see Fig. 6d).

It is noted that the vessel intensity of the flow-sensitive PC-MRA images fluctuates significantly because of the variation of blood flow speeds inside the vessels with different sizes. This intensity fluctuation produces image gradient along vascular structures and stops the evolving contours of CURVES inside the vessels (see Figs. 7f and b). For FLUX, the faint responses detected by the symmetric discretized Laplacian operator cannot precisely position the boundaries of the vessels. The evolving contours leak through the object boundaries and are subsequently guided by image noise as shown in Figs. 7r and d. In TOF-MRA image, the non-vascular tissues can report intensity values similar to those of vascular regions (see Fig 7k, right). It greatly reduces the intensity contrast of the vessel boundaries where a non-vascular structure with similar intensity is nearby. As a result, the weak vessel boundaries cannot exert enough image force to draw the evolving contours of CURVES along the vessels and causes under-segmentation (Fig. 7 ). In Fig. 7g, the contour of FLUX expands beyond the weak vessel boundaries and follows the non-vascular structures. In the CTA image, the evolving contours of both CURVES and FLUX (Figs.7f and h) leak through the arteries and follow the edges of the heart chamber surface. The contour evolution of FLUX and CURVES in this case was manually stopped for contour visualization.

In contrast, the measure $M(\boldsymbol{x})$ of the proposed method encourages contours to expand along vessels despite the intensity variation of vessels. On the other hand, the OFA based measure, as stated in Equation 7 is capable of halting the evolving contours at the vessel boundaries. It can segment the vessels without leakage (Figs. [8 $\mathrm{A}-\mathrm{c}$ ). Based on the visual comparison between the segmented vessels of the proposed method, and the original image volumes shown in Figs. 6a-c, the proposed method is able to deliver faithful segmentation results. It can also withstand the disturbance introduced by the irrelevant edges adjacent to the target structures. Thus, the proposed method successfully segment the coronary arteries as presented in Fig. $8 \mathrm{~d}$.

\section{Perspective and Conclusion}

The proposed active contour model is devised based on various measures which aim at locally quantifying the image gradient symmetry. In our application vascular segmentation, since the tissue intensity can vary spatially due to the presence of multiplicative bias field, the proposed model avoids encapsulating the regional intensity variance information [2][5]. Albeit the three dimensional formulation of the proposed method, it is general to cope with curvilinear structures in two, three or higher dimensions, if any. Also, we are acquiring more data sets and also segmenting ground truth in order to perform quantitative comparison to other approaches.

Besides, analogous to the studies in [8][10], introducing geometric constraints to the proposed active contour model may be beneficial. The major concern is that these geometric constraints require intensive parameter searching. Furthermore, vast numbers of application specific constraints or supplementary information have been proposed recently, for instance, detecting only structures with circular cross sections, regularization based on the curvature of structure centerlines or structure radii, disallowing bifurcation, 
exploiting training data or interactive segmentation (see [15] for a comprehensive survey). The proposed measures can provide useful features to detect curvilinear structures along with these constraints or supplementary information for particular applications.

Regarding the proposed active contour model, the oriented flux symmetry based formulation expands the evolving contours in the middle of curvilinear structures where the image gradients are symmetric. The contours are eventually driven to the object boundaries, in order to maximize the gradient antisymmetry along the contour inward normal direction. Benefited from the oriented flux antisymmetry measure and the oriented flux measure, the proposed model is capable of segmenting the entire structures without contour leakages, in both the experiments using the synthetic image and the real images of different modalities. It is experimentally demonstrated that the oriented flux symmetry based active contour model achieves promising segmentation results.

\section{References}

1. Caselles, V., et al.: Geodesic active contours. IJCV 22(1), 61-79 (1997)

2. Chan, T., Vese, L.: Active contours without edges. TIP 10(2), 266-277 (2001)

3. Descoteaux, M., Collins, L., Siddiqi, K.: Geometric flows for segmenting vasculature in MRI: Theory and validation. In: Barillot, C., Haynor, D.R., Hellier, P. (eds.) MICCAI 2004. LNCS, vol. 3216, pp. 500-507. Springer, Heidelberg (2004)

4. Frangi, A.F., Niessen, W.J., Vincken, K.L., Viergever, M.A.: Multiscale vessel enhancement filtering. In: Wells, W.M., Colchester, A.C.F., Delp, S.L. (eds.) MICCAI 1998. LNCS, vol. 1496, p. 130. Springer, Heidelberg (1998)

5. Gooya, A., Liao, H., Matsumiya, K., Masamune, K., Dohi, T.: Effective statistical edge integration using a flux maximizing scheme for volumetric vascular segmentation in MRA. In: Karssemeijer, N., Lelieveldt, B. (eds.) IPMI 2007. LNCS, vol. 4584, pp. 86-97. Springer, Heidelberg (2007)

6. Koller, T., et al.: Multiscale detection of curvilinear structures in 2-d and 3-d image data. In: ICCV, pp. 864-869 (1995)

7. Law, M., Chung, A.: Three dimensional curvilinear structure detection using optimally oriented flux. In: Forsyth, D., Torr, P., Zisserman, A. (eds.) ECCV 2008, Part IV. LNCS, vol. 5305, pp. 368-382. Springer, Heidelberg (2008)

8. Lorigo, L., et al.: Curves: Curve evolution for vessel segmentation. MedIA 5(3), 195-206 (2001)

9. Osher, S., Sethian, J.: Fronts propagating with curvature dependent speed: Algorithms based on hamilton-jacobi formulations. J. Comp. Phys. 79(1), 12-49 (1988)

10. Sato, Y., et al.: Three-dimensional multi-scale line filter for segmentation and visualization of curvilinear structures in medical images. MedIA 2(2), 143-168 (1998)

11. Toledo, R., et al.: Eigensnakes for vessel segmentation in angiography. In: ICPR, pp. 4340 4343 (2000)

12. Vasilevskiy, A., Siddiqi, K.: Flux maximizing geometric flows. PAMI 24(12), 1565-1578 (2002)

13. Whitaker, R.: A level-set approach to 3d reconstruction from range data. IJCV 29(33), 203231 (1998)

14. Yan, P., Kassim, A.: Segmentation of volumetric mra images by using capillary active contour. MedIA 10(3), 317-329 (2006)

15. Lesage, D., et al.: A review of $3 \mathrm{D}$ vessel lumen segmentation techniques: Models, features and extraction schemes. MedIA 13(6), 819-845 (2009) 